\title{
A Fuzzy Knowledge-based Decision Support System for Tender Call Evaluation
}

\author{
Panos Alexopoulos, Manolis Wallace, Konstantinos Kafentzis, and Aristodimos \\ Thomopoulos
}

\begin{abstract}
In the modern business environment, the capability of an enterprise to generate value from its business knowledge influences in an increasingly important way its competitiveness. Towards this direction, knowledge-based systems can be a very effective tool for enhancing the productivity of knowledge workers by providing them with advanced knowledge processing capabilities. In this paper we describe such a system which utilizes organizational and domain knowledge in order to support consultants in the process of evaluating calls for tender.
\end{abstract}

\section{Introduction}

In the modern business environment, the capability of an enterprise to generate value from its business knowledge influences in an increasingly important way its competitiveness [2]. In fact, in the knowledge management terminology, the term "knowledge asset" is used for denoting enterprise knowledge that regards markets, products, technologies and organisations and which enables the enterprise's business processes to generate profits and value [4].

The techniques to manage knowledge assets are drawn from two distinct areas: traditional business management and knowledge-based systems. The latter are software applications that are able to emulate the work of experts in specific areas of

Panos Alexopoulos $\cdot$ Konstantinos Kafetzis

IMC Technologies S.A., Fokidos 47, 11527, Athens, Greece,

e-mail: \{palexopoulos, kkafentzis\}@imc.com.gr

Manolis Wallace

Department of Computer Science and Technology University of Peloponnese, End of Karaiskaki St., 22100, Tripolis, Greece, e-mail: wallace@uop.gr

Aristodimos Thomopoulos

DIADIKASIA S.A., 180 Kifissias Av.,152 31, Halandri, Athens, Greece,

e-mail: athomopoulos@diadikasia.gr

Please use the following format when citing this chapter:

Alexopoulos, P., Wallace, M., Kafentzis, K. and Thomopoulos, A., 2009, in IFIP International Federation for Information Processing, Volume 296; Artificial Intelligence Applications and Innovations III; Eds. Iliadis, L., Vlahavas, I., Bramer, M.; (Boston: Springer), pp. 51-59. 
knowledge. As such they can be a very effective tool for enhancing the productivity of knowledge workers who are, after all, some of the most important knowledge assets of an enterprise.

In this paper we describe a knowledge-based system that is used by consulting firms for tackling the problem of the evaluation of tender calls. A tender call is an open request made by some organization for a written offer concerning the procurement of goods or services at a specified cost or rate. The evaluation of a (consulting related) tender call by a consulting company refers to the process of deciding whether the company should devote resources for preparing a competitive tender in order to be awarded the bid.

In our approach, we formulate the above problem as a decision making problem in which the decision to be taken is whether the company should write the tender or not. The evaluation criterion is the probability that the company will be assigned the call's project. This is usually estimated by some consultant who takes in mind a number of different (and often conflicting) factors (criteria) that, according to his/her judgement and experience, influence it. These factors can be classified into objective and subjective but, most importantly, the whole process of evaluating and combining all those factors is knowledge-intensive. And that is because the consultant utilizes during this process aspects of the company's organizational knowledge as well as knowledge derived from his/her own expertise.

For that, the key characteristic of our system is that it stores and utilizes all the necessary knowledge that the consultant needs for taking an informed decision and performs reasoning that is very close to the his/her way of thinking. Furthermore, both the reasoning and the underlying knowledge have fuzzy characteristics and that is because many aspects of the knowledge the consultant utilizes for his/her evaluation are inherently imprecise.

The structure of the rest of the paper is as follows: In section 2 we outline some of the key criteria that the consultant uses for evaluating the partial factors that influence the company's chances to win the tender should a proposal be written. For each criterion, we highlight the knowledge that is required for its evaluation. In section 3 we describe the system's knowledge base which consists of a fuzzy domain ontology while in section 4 we present the reasoning mechanism in the form of fuzzy reasoning procedures for each criterion. Finally, in section 5 we provide a representative use case of the system and in section 6 we conclude by addressing issues for future work.

\section{Tender Call Evaluation Criteria}

The tender call evaluation process that our system implements comprises the evaluation of a number of partial criteria such as the call's budget, the call's coverage by the consulting company's expertise and experience and the potential competition. Due to space limitations, we describe here only two of the above criteria which, 
nevertheless, are quite representative of our approach. Along with the descriptions, we highlight the kind of knowledge required for the evaluation of each criterion.

\subsection{Call's Relevance to the Consulting Company's Expertise}

Any consultant or consulting company has expertise in specific business or technology areas and thus calls for tender which address topics outside of these areas are usually not pursued. This happens not only because the chances of winning such a call are significantly slimmer but also because, even if the call is won, the project's cost and failure probability will be very high. Thus, an important evaluation criterion is the degree at which the call's addressed topics are satisfied by the consultant's expertise.

The knowledge the consultant utilizes for the estimation of this degree includes first of all the list of areas at which the company is expert. Furthermore, he/she is able to determine whether two different areas are similar or not. For example, if the call is about a marketing project and the company is expert at sales support projects, then the two areas are quite similar. On the other hand, if the call's project is about developing an ontology-based system and the company does not know how to develop ontologies then there is no point at writing a tender for this call.

In any case, the ability to evaluate and quantify the similarity between two areas is of essential importance in deciding whether an effort should be made to address a tender call. As a consequence, the automated estimation of such degrees of relevance is a central part of this work and of our system.

\subsection{Call's Coverage by the Consulting Company's Experience}

The experience of a consulting company is usually measured by the number of the projects it has successfully implemented and delivered in the past. A large number of successful projects in some specific business area denotes a high level of experience in this area and conversely. And high level of experience in a tender call's addressed business areas is highly desirable for two reasons: i) it provides proof of the consulting company's ability to successfully handle projects similar to the requested and ii) the know-how gained through this experience makes the implementation of the requested project less costly and less risky.

In order to measure the relevant experience of the company, the consultant needs not only to know of all the projects the company has already implemented, but also and to be able to evaluate and quantify their relevance to the call. 


\section{System's Knowledge Base}

The knowledge base of the system is implemented as a Fuzzy Ontology [1]. A Fuzzy Ontology is a tuple $\mathbf{O}_{F}=\{\mathbf{E}, \mathbf{R}\}$ where $\mathbf{E}$ is a set of semantic entities (or concepts) and $\mathbf{R}$ is a set of fuzzy binary semantic relations. Each element of $\mathbf{R}$ is a function $R: \mathbf{E}^{2} \rightarrow[0,1]$.

In particular, $\mathbf{R}=\mathbf{T} \cup \mathbf{N T}$ where $\mathbf{T}$ is the set of taxonomic relations and $\mathbf{N T}$ is the set of non-taxonomic relations. Fuzziness in a taxonomic relation $R \in \mathbf{T}$ has the following meaning: High values of $R(a, b)$, where $a, b \in E$, imply that $b$ 's meaning approaches that of $a$ 's while low values suggest that $b$ 's meaning becomes "narrower" than that of $a$ 's.

On the other hand, a non-taxonomic relation has an ad-hoc meaning defined by the ontology engineer. Fuzziness in this case is needed when such a relation represents a concept for which there is no exact definition. In that case fuzziness reflects the degree at which the relation can be considered as true.

In our case, the structure of the system's ontology is dictated by the knowledge requirements identified in the previous section. Thus, the ontology consists of three groups of concepts, namely Companies, Projects and ConsultingAreas. The relation between different consulting areas is represented by means of a taxonomical relation called hasSubArea. The areas at which a company considers itself expert, are captured through the fuzzy non-taxonomical relation isExpertAt while the areas a project is relevant to are represented through the fuzzy non-taxonomical relation isRelevantTo. Finally, the projects a company has implemented or participated in are denoted by the (non-fuzzy) relation hasImplemented. A snapshot of the consulting areas taxonomy is shown in figure 1 .

\section{Evaluation of Criteria}

\subsection{Call's Relevance to the Consultant's Expertise}

As mentioned in paragraph 2.1, the consultant assesses the call's relevance to the company's expertise by comparing the corresponding consulting areas. Consequently, such a comparison will need to be performed by the decision support system. For that there are three requirements:

1. The system needs to know the company's areas of expertise.

2. The system needs to know the areas covered by the call.

3. The system needs to have an explicit mechanism for determining the degree to which the call's areas are covered by the company's ones.

Requirement 2 is the most interesting one from a research point of view. In this work the automated estimation of the thematic coverage of the call is performed via 


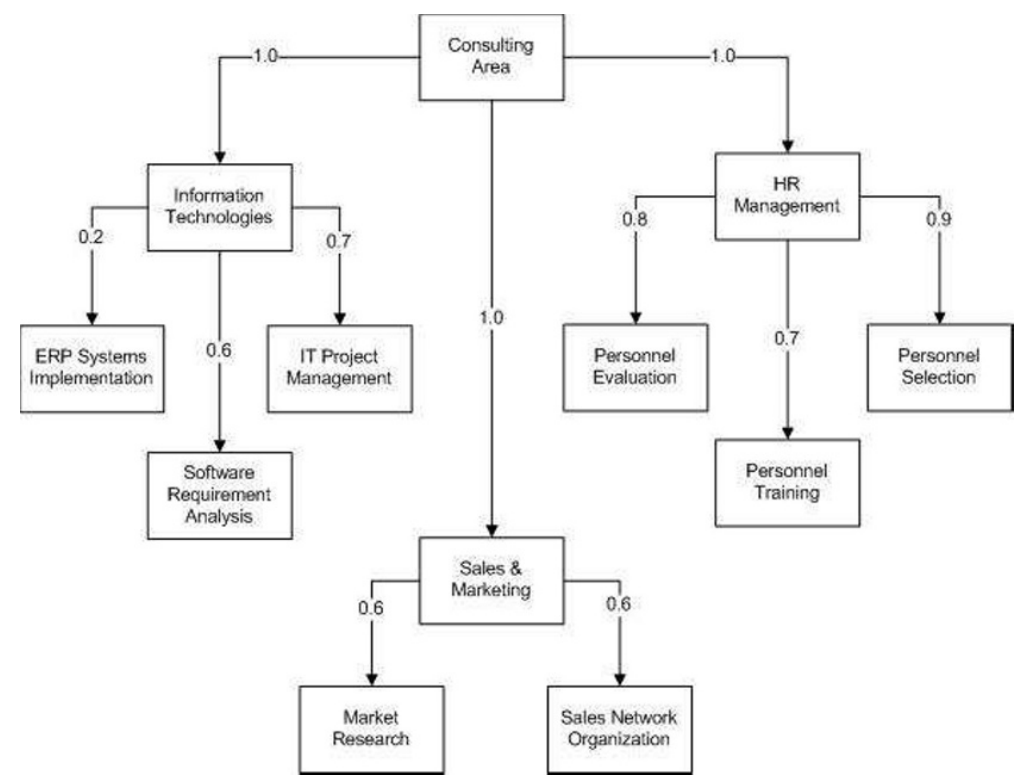

Fig. 1 Consulting Areas Fuzzy Taxonomy

a properly adapted version of the Detection of Thematic Categories (DTC) algorithm [6].

The DTC algorithm processes free text and extracts the fuzzy set of thematic categories that are associated with it via the exploitation of information contained in a fuzzy relational representation. With a small adaptation, the DTC algorithm is used to process the text of a call considering the system's knowledge base as input knowledge, thus generating a fuzzy set that contains the call's consulting areas and their associated degrees.

More specifically, the adapted DTC algorithm identifies in the call's text all terms mentioned in the system's knowledge base and then clusters them using an agglomerative approach; the inter-cluster distance measure used in the agglomeration is based on the notion of context [5], which measures how semantically close two concepts are, based on their distance in the fuzzy relations that comprise the system's knowledge. The thematic content and fuzzy cardinality of the resulting clusters provide the areas and their degrees respectively.

For requirement 1, namely the company's areas of expertise, the system utilizes the fuzzy relation isExpertAt from the ontology and retrieves a fuzzy set that contains the consulting areas and their associated degrees. This fuzzy set is then processed by the DTC algorithm and the result is a new fuzzy set that contains the thematic categories of the company's areas of expertise.

Given the above sets, namely the thematic categories of the areas in which the company has expertise and the set of areas relevant to the call, the call's coverage by the consulting company is calculated as follows, thus addressing requirement 3 : 


$$
\text { Coverage }=\frac{h(\text { CallThematicContent } \cap \text { CompanyExpertiseThematicContent })}{h(\text { CallThematicContent })}
$$

where $h(A)$ denotes the height of the fuzzy set $A$ and the conjunction operation is performed by means of the min t-norm [3].

\subsection{Company's Experience}

The company's experience in a certain consulting area depends on the number of relevant projects already implemented. Thus, in order for the system to assess the degree to which the call's requirements are covered against the overall company's experience, it needs to measure first the relevance of each of the company's implemented projects to the call and then calculate an overall coverage measure. For that, the following steps are followed:

1. The call's consulting areas are extracted into a fuzzy set as explained in paragraph 4.1

2. The company's implemented projects are retrieved from the ontology as fuzzy sets.

3. The relevance of each past project to the call is calculated, also as explained in paragraph 4.1

4. Past projects whose relevance is less than a specific threshold (defined by the consultant) are discarded. For the remaining projects we calculate their accumulated relevance to the call.

5. The overall relevance is calculated by applying to the accumulated a fuzzy triangular number of the form $(0, a, \infty)$

6. The parameter $a$ denotes the threshold over which additional past project do not further influence the overall similarity.

\subsection{Overall Evaluation}

For the overall evaluation of a tender call we use a technique based on the notion of the ordered weighted averaging operator(OWA), first introduced by Yager in [8]. An OWA operator of dimension $n$ is a mapping $F: R^{n} \rightarrow R$ that has an associated $n$ vector $w=\left(w_{1}, w_{2}, \ldots, w_{n}\right)^{T}$ such as $w_{i} \in[0,1], 1 \leq i \leq n$ and $\sum_{j=1}^{n} w_{i}=1$. Given this operator the aggregated value of a number of decision criteria ratings is $F\left(a_{1}, a_{2}, \ldots, a_{n}\right)=\sum_{j=1}^{n} w_{j} b_{j}$ where $b_{j}$ is the $\mathrm{j}$-th largest element of the bag $<a_{1}, a_{2}, \ldots, a_{n}>$.

The fundamental aspect of OWA operators is the fact that an aggregate $a_{i}$ is not associated with a particular weight $w_{i}$ but rather a weight is associated with a partic- 
ular ordered position of the aggregate. This, in our case, is useful as most consultants are not able to define a-priori weights to criteria since, as they say, the low rating of a criterion might be compensated by the high rating of another criterion.

OWA operators can provide any level of criteria compensation lying between the logical and and or. Full compensation (or) is implemented through the operator $w=(1,0, \ldots, 0)^{T}$ while zero compensation through the operator $w=(0,0, \ldots, 1)^{T}$. In our case the compensation level is somewhat above average. This was determined with the help of domain experts consultants after several trials of the system.

\section{Use Case}

The testing and evaluation of the effectiveness of our system was conducted by DIADIKASIA S.A., a Greek consulting firm that provides a wide range of specialized services to organizations and companies of both the public and private sector. The whole process comprised two steps, namely the population of the system's ontology with company specific knowledge and the system's usage by the company's consultants for evaluating real tender calls.

For the first step, instances of the relations isExpertAt and hasImplemented were generated by the consulting company while the population of the relation isRelevantTo was performed automatically by applying the DTC algorithm to the company's past projects' descriptions. Thus, considering the areas taxonomy of figure 1, a part of the ontology for DIADIKASIA was as follows:

- $\quad$ isExpertAt(DIADIKASIA, Software Requirement Analysis)=0.6

- $\quad$ isExpertAt(DIADIKASIA, IT Project Management) $=0.8$

- hasImplemented(DIADIKASIA, EDRASI)

- isRelevantTo(EDRASI, Information Technologies) $=0.8$

For the second step, the company's consultants used the system for evaluating real tender calls. Figure 2 illustrates an evaluation scenario in which of tender call entitled "Requirement Analysis for the ERP System of company X". As it can be seen in the figure, the system identifies, through the DTC algorithm, the call's area as "Information Technology" at a degree of 0.77 and evaluates the expertise and the experience of the company with scores $77 \%$ and $25 \%$ respectively. The overall evaluation given these two criteria and through the OWA operator is $60 \%$.

\section{Conclusions \& Future Work}

In this paper we described a knowledge-based decision support system that is used by consulting firms for tackling the problem of the evaluation of tender calls. The key characteristic of our system is that it applies fuzzy reasoning over organizational 


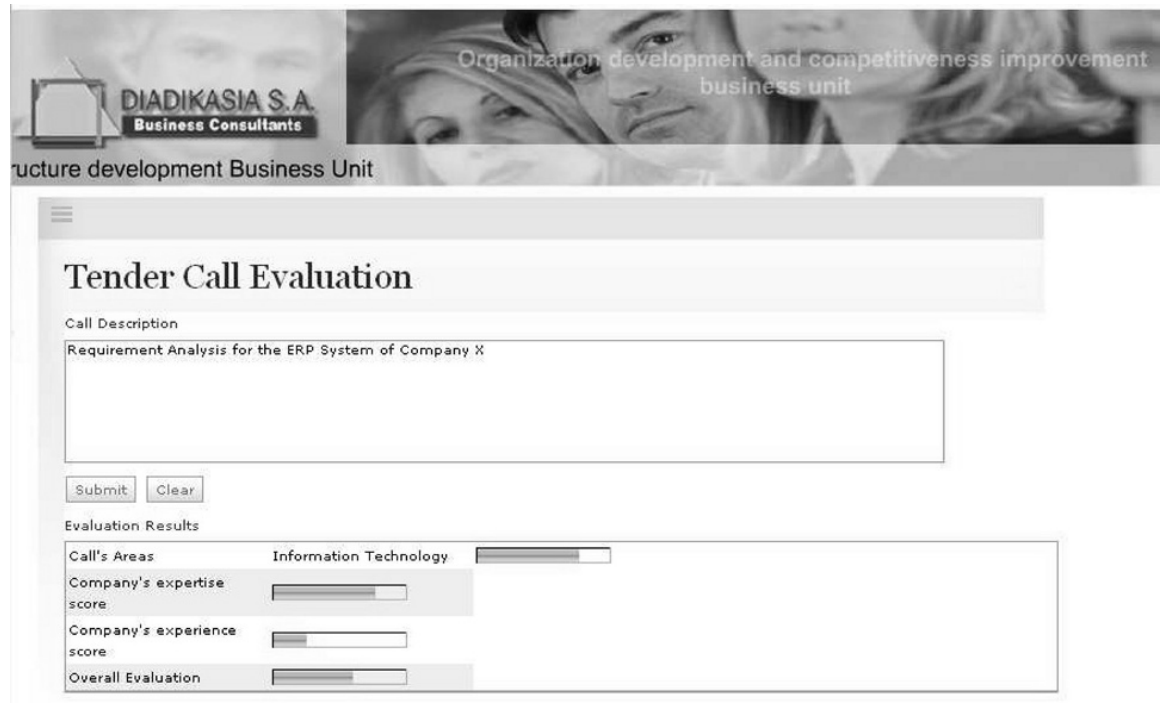

(2) 2008 Diadikasia S.A.

Fig. 2 Evaluation Scenario

and domain specific knowledge in order to model as accurately and as intuitively as possible the consultant's way of thinking.

The work and sub-system presented here in are part of a larger work and integrated system, also comprising the methodology and subsystem to support the consultant in the actual preparation of the proposal text, in the case that the overall evaluation leads to a decision to address the call. This second sub-system is also based mainly on the fuzzy ontology and the DTC algorithm in order to analyze existing texts and estimate which are most relevant and potentially useful inputs in this process. The sub-system supporting the preparation of the proposal, together with the relevant theory and methodologies, fall beyond the scope of the present paper but will be presented in detail in a future work.

\section{References}

1. Calegari, S., and Sanchez, E. A Fuzzy Ontology-Approach to improve Semantic Information Retrieval. In Bobillo, F., da Costa, P.C.G., D’Amato, C., Fanizzi, N., Fung, F., Lukasiewicz, T., Martin, T., Nickles, M., Peng, Y., Pool, M., Smrz, P., Vojtas, P., eds.: Proceedings of the Third ISWC Workshop on Uncertainty Reasoning for the Semantic Web - URSW'07. Volume 327 of CEUR Workshop Proceedings., CEUR-WS.org (2007).

2. Drucker, P.F. "The Coming of the New Organization" in Harvard Business Review on Knowledge Management. Harvard Business School Press, 1998. pp. 1-19. 
3. Klir, G., Yuan, B. (1995) Fuzzy Sets and Fuzzy Logic, Theory and Applications. Prentice Hall.

4. Mentzas, G., Apostolou, D., Abecker, A., Young, R., 2002, "Knowledge Asset Management: Beyond the Process-centred and Product-centred Approaches", Series: Advanced Information and Knowledge Processing, Springer London.

5. Wallace, M., Avrithis, Y., "Fuzzy Relational Knowledge Representation and Context in the Service of Semantic Information Retrieval", Proceedings of the IEEE International Conference on Fuzzy Systems (FUZZ-IEEE), Budapest, Hungary, July 2004

6. Wallace, M., Mylonas, Ph., Akrivas, G., Avrithis, Y. \& Kollias, S., "Automatic thematic categorization of multimedia documents using ontological information and fuzzy algebra", in Ma, Z. (Ed.), Studies in Fuzziness and Soft Computing, Soft Computing in Ontologies and Semantic Web, Springer, Vol. 204, 2006.

7. Yager, R.R. "On ordered weighted averaging aggregation operators in multi-criteria decision making”, IEEE Transactions on Systems, Man and Cybernetics 18(1988) 183-190. 